\title{
Solutions of nonlinear systems by reproducing kernel method
}

\author{
Ali Akgüla,*, Yasir Khan ${ }^{b}$, Esra Karatas Akgül ${ }^{\mathrm{C}}$, Dumitru Baleanu ${ }^{\mathrm{d}, \mathrm{e}}$, Maysaa Mohamed Al Qurashi ${ }^{\mathrm{f}}$ \\ ${ }^{a}$ Department of Mathematics, Art and Science Faculty, Siirt University, 56100 Siirt, Turkey. \\ ${ }^{b}$ Department of Mathematics, University of Hafr Al-Batin, Hafr Al-Batin 31991, Saudi Arabia. \\ ${ }^{c}$ Gelibolu Piri Reis Vocational School, Çanakkale Onsekiz Mart University, 17100, Çanakkale, Turkey. \\ ${ }^{d}$ Department of Mathematics and Computer Sciences, Art and Science Faculty, Çankaya University, TR-06300 Ankara, Turkey. \\ ${ }^{e}$ Department of Mathematics, Institute of Space Sciences, Bucharest, Romania. \\ ${ }^{f}$ Department of Mathematics, King Saud University, Riyadh 12364, Saudi Arabia.
}

Communicated by A. Atangana

\begin{abstract}
A novel approximate solution is obtained for viscoelastic fluid model by reproducing kernel method (RKM). The resulting equation for viscoelastic fluid with magneto-hydrodynamic flow is transformed to the nonlinear system by introducing the dimensionless variables. Results are presented graphically to study the efficiency and accuracy of the reproducing kernel method. Results show that this method namely RKM is an efficient method for solving nonlinear system in any engineering field. (C)2017 All rights reserved.
\end{abstract}

Keywords: Reproducing kernel method, series solutions, nonlinear systems. 2010 MSC: 47B32, 46E22, 35M32, 74S30.

\section{Introduction}

We conceive the following nonlinear system in this work [29]:

$$
\left\{\begin{array}{l}
z^{(v)}+a_{0}(\varpi) z^{(i v)}+a_{1}(\varpi) z^{\prime \prime \prime}+a_{2}(\varpi) z^{\prime \prime}+a_{3}(\varpi) z^{\prime}+a_{4}(\tau) z \\
\quad+s^{(v)}+a_{5}(\varpi) s^{(i v)}+a_{6}(\varpi) s^{\prime \prime \prime}+a_{7}(\varpi) s^{\prime \prime}+a_{8}(\varpi) s^{\prime}+a_{9}(\varpi) s+M_{1}(z, s)=0, \\
z^{(v)}+b_{0}(\varpi) z^{(i v)}+b_{1}(\varpi) z^{\prime \prime \prime}+b_{2}(\varpi) z^{\prime \prime}+b_{3}(\varpi) z^{\prime}+b_{4}(\varpi) z \\
\quad+s^{(v)}+b_{5}(\varpi) s^{(i v)}+b_{6}(\varpi) s^{\prime \prime \prime}+b_{7}(\varpi) s^{\prime \prime}+b_{8}(\varpi) s^{\prime}+b_{9}(\varpi) s+M_{2}(z, s)=0, \\
z=0, \quad z^{\prime}=0, \quad s=0, \quad \text { at } \quad \varpi=-1, \\
z=0, \quad z^{\prime}=0, \quad s=0, \quad \text { at } \quad \varpi=1,
\end{array}\right.
$$

where $M_{1}$ and $M_{2}$ are nonlinear functions of $z, s$, and $a_{j}(\varpi), b_{j}(\varpi)$ are continuous, $j=0,1, \ldots, 9$. Ordinary differential systems are significant for real-world problems. These systems have been implemented to many problems [8-10, 17]. Biswas et al. studied systems by many different methods $[11,15,20]$.

\footnotetext{
*Corresponding author

Email addresses: aliakgul00727@gmail.com (Ali Akgül), yasirmath@yahoo.com (Yasir Khan), esrakaratas@comu.edu.tr (Esra Karatas Akgül), Maysaa@ksu.edu.sa (Maysaa Mohamed Al Qurashi)

doi:10.22436/jnsa.010.08.33
} 
In this work, we demonstrate the solutions of (1.1). We suppose that (1.1) has a unique solution. Put

$$
\left\{\begin{array}{l}
\mathrm{B}_{11} z=z^{(v)}+\mathrm{a}_{0}(\varpi) z^{(i v)}+\mathrm{a}_{1}(\varpi) z^{\prime \prime \prime}+\mathrm{a}_{2}(\varpi) z^{\prime \prime}+\mathrm{a}_{3}(\varpi) z^{\prime}+a_{4}(\varpi) z \\
\mathrm{~B}_{12} s=s^{(v)}+\mathrm{a}_{5}(\varpi) s^{(i v)}+\mathrm{a}_{6}(\varpi) s^{\prime \prime \prime}+\mathrm{a}_{7}(\varpi) s^{\prime \prime}+\mathrm{a}_{8}(\varpi) s^{\prime}+\mathrm{a}_{9}(\varpi) s \\
\mathrm{~B}_{21} z=z^{(v)}+b_{0}(\varpi) z^{(i v)}+b_{1}(\varpi) z^{\prime \prime \prime}+b_{2}(\varpi) z^{\prime \prime}+b_{3}(\varpi) z^{\prime}+b_{4}(\varpi) z \\
\mathrm{~B}_{22} s=s^{(v)}+b_{5}(\varpi) s^{(i v)}+b_{6}(\varpi) s^{\prime \prime \prime}+b_{7}(\varpi) s^{\prime \prime}+b_{8}(\varpi) s^{\prime}+b_{9}(\varpi) s \\
B=\left(\begin{array}{ll}
B_{11} & B_{12} \\
B_{21} & B_{22}
\end{array}\right) \text { and } v=(z, s)^{\top}
\end{array}\right.
$$

then (1.1) can be written as:

$$
\left\{\begin{array}{l}
\mathrm{L} v=\mathrm{M}(z, \mathrm{~s}), \quad-1 \leqslant \varpi \leqslant 1, \\
v(-1)=0=v(1)
\end{array}\right.
$$

where $M=\left(M_{1}, M_{2}\right)^{\top}, v \in \widehat{W}_{2}^{6}[-1,1] \oplus \widehat{W}_{2}^{6}[-1,1], M \in \widehat{W}_{2}^{1}[-1,1] \oplus \widehat{W}_{2}^{1}[-1,1]$. The space

$$
\widehat{W}_{2}^{6}[-1,1] \oplus \widehat{W}_{2}^{6}[-1,1],
$$

is defined as

$$
\widehat{W}_{2}^{6}[-1,1] \oplus \widehat{W}_{2}^{6}[-1,1]=\left\{v=(z, s)^{\top} \mid h, v \in \widehat{W}_{2}^{6}[-1,1]\right\} .
$$

The inner product and norm are presented as

$$
\langle v, u\rangle=\sum_{i=1}^{2}\left\langle v_{i}, u_{i}\right\rangle_{\widehat{W}_{2}^{6}}, \quad\|v\|=\left(\sum_{i=1}^{2}\left\|v_{i}\right\|^{2}\right)^{\frac{1}{2}}, \quad v, u \in \widehat{W}_{2}^{6}[-1,1] \oplus \widehat{W}_{2}^{6}[-1,1] .
$$

$\widehat{W}_{2}^{6}[-1,1] \oplus \widehat{W}_{2}^{6}[-1,1]$ is a reproducing kernel space. $\widehat{W}_{2}^{1}[-1,1] \oplus \widehat{W}_{2}^{1}[-1,1]$ may be described in a similar way.

The theory of reproducing kernels [5] was utilized for the first time by Zaremba. Reproducing kernel theory has significant implementations in numerical analysis, differential equations, probability and statistics [18, 35, 37, 40]. Recently, utilizing the reproducing kernel method (RKM), some authors investigated fractional differential equations, nonlinear oscillators with discontinuity, singular and nonlinear partial differential equations [1-4, 6, 7, 12-14, 16, 19, 21-28, 30-34, 36, 38, 39, 41].

This paper is prepared as follows. Section 2 presents several useful reproducing kernel Hilbert spaces and reproducing kernel functions. The representation in $\widehat{W}_{2}^{6}[-1,1] \oplus \widehat{W}_{2}^{6}[-1,1]$ and a related linear operator are given in Section 3. This section gives the main results. Numerical examples are shown in Section 4. The final section contains some conclusions.

\section{Reproducing kernel spaces and their reproducing kernel functions}

Definition 2.1. We give the space $\widehat{W}_{2}^{6}[-1,1]$ as

$$
\begin{gathered}
\widehat{\mathrm{W}}_{2}^{6}[-1,1]=\left\{v \in \mathrm{AC}[-1,1]: v^{\prime}, v^{\prime \prime}, v^{(3)}, v^{(4)}, v^{(5)} \in \mathrm{AC}[-1,1], v^{(6)} \in \mathrm{L}^{2}[-1,1],\right. \\
\left.v(-1)=v^{\prime}(1)=v^{\prime}(-1)=v^{\prime}(1)=0\right\} .
\end{gathered}
$$


The inner product and the norm in $\widehat{W}_{2}^{5}[-1,1]$ are given as

$$
\langle v, u\rangle_{\widehat{W}_{2}^{6}}=\sum_{i=0}^{5} v^{(i)}(0) u^{(i)}(0)+\int_{0}^{1} v^{(6)}(x) u^{(6)}(x) d x, \quad v, u \in \widehat{W}_{2}^{6}[-1,1],
$$

and

$$
\|v\|_{\widehat{W}_{2}^{6}}=\sqrt{\left\langle v, v \widehat{W}_{2}^{6}\right.}, \quad v \in \widehat{W}_{2}^{6}[-1,1]
$$

Theorem 2.2. Reproducing kernel function $\widetilde{\mathrm{R}_{\mathrm{y}}}$ of $\widehat{\mathrm{W}}_{2}^{6}[-1,1]$ is obtained as:

$$
\widetilde{R_{y}}(x)= \begin{cases}\sum_{i=1}^{12} c_{i}(y) x^{i-1}, & x \leqslant y \\ \sum_{i=1}^{12} d_{i}(y) x^{i-1}, & x>y\end{cases}
$$

Proof. By Definition 2.1, we have

$$
\left\langle v, \widetilde{R_{y}}\right\rangle_{\widehat{W}_{2}^{6}}=\sum_{i=0}^{5} v^{(i)}(-1){\widetilde{R_{y}}}^{(i)}(-1)+\int_{-1}^{1} v^{(6)}(x){\widetilde{R_{y}}}^{(6)}(x) \mathrm{d} x, \quad v, \widetilde{R_{y}} \in \widehat{W}_{2}^{6}[-1,1] .
$$

Integrating this equation by parts, we have

$$
\begin{aligned}
\left\langle v, \widetilde{R_{y}}\right\rangle_{\widetilde{W}_{2}^{6}}= & v(-1) \widetilde{R_{y}}(-1)+v^{\prime}(-1){\widetilde{R_{y}}}^{\prime}(-1)+v^{\prime \prime}(-1){\widetilde{R_{y}}}^{\prime \prime}(-1) \\
& +v^{(3)}(-1){\widetilde{R_{y}}}^{(3)}(-1)+v^{(4)}(-1){\widetilde{R_{y}}}^{(4)}(-1)+v^{(5)}(-1){\widetilde{R_{y}}}^{(5)}(-1) \\
& +v^{(5)}(1){\widetilde{R_{y}}}^{(6)}(1)-v^{(5)}(-1){\widetilde{R_{y}}}^{(6)}(-1)-v^{(4)}(1){\widetilde{R_{y}}}^{(7)}(1) \\
& +v^{(4)}(-1){\widetilde{R_{y}}}^{(7)}(-1)+v^{\prime \prime \prime}(1){\widetilde{R_{y}}}^{(8)}(1)-v^{\prime \prime \prime}(-1){\widetilde{R_{y}}}^{(8)}(-1) \\
& -u^{\prime \prime}(1){\widetilde{R_{y}}}^{(9)}(1)+v^{\prime \prime}(-1){\widetilde{R_{y}}}^{(9)}(-1)+v^{\prime}(1){\widetilde{R_{y}}}^{(10)}(1) \\
& -v^{\prime}(-1){\widetilde{R_{y}}}^{(10)}(-1)-v(1){\widetilde{R_{y}}}^{(11)}(1) \\
& +v(-1){\widetilde{R_{y}}}^{(11)}(-1)+\int_{-1}^{1} v(x){\widetilde{R_{y}}}^{(12)}(x) \mathrm{d} x .
\end{aligned}
$$

By reproducing property, we have

$$
\left\langle v, \widetilde{R_{y}}\right\rangle_{\widehat{W}_{2}^{6}}=v(y)
$$

Since $\widetilde{R_{y}} \in \widehat{W}_{2}^{6}[-1,1]$, we get

$$
\widetilde{R_{y}}(-1)=R_{y}^{\prime}(-1)=\widetilde{R_{y}}(1)=R_{y}^{\prime}(1)=0
$$

If

$$
\left\{\begin{array}{l}
{\widetilde{R_{y}}}^{\prime \prime}(-1)+{\widetilde{R_{y}}}^{(9)}(-1)=0, \\
{\widetilde{R_{y}}}^{(3)}(-1)-{\widetilde{R_{y}}}^{(8)}(-1)=0, \\
{\widetilde{R_{y}}}^{(4)}(-1)+{\widetilde{R_{y}}}^{(7)}(-1)=0, \\
{\widetilde{R_{y}}}^{(5)}(-1)-{\widetilde{R_{y}}}^{(6)}(-1)=0, \\
{\widetilde{R_{y}}}^{(6)}(1)=0, \\
{\widetilde{R_{y}}}^{(7)}(1)=0, \\
{\widetilde{R_{y}}}^{(8)}(1)=0, \\
\widetilde{{\widetilde{R_{y}}}^{(9)}}(1)=0,
\end{array}\right.
$$


then (2.1) gives

$$
{\widetilde{R_{y}}}^{(12)}(x)=\delta(x-y) .
$$

When $x \neq y$,

$$
\widetilde{R}^{(12)}(x)=0,
$$

therefore

$$
\widetilde{R_{y}}(x)= \begin{cases}\sum_{i=1}^{12} c_{i}(y) x^{i-1}, & x \leqslant y, \\ \sum_{i=1}^{12} d_{i}(y) x^{i-1}, & x>y .\end{cases}
$$

Since

$$
{\widetilde{R_{y}}}^{(12)}(x)=\delta(x-y),
$$

we get

$$
\partial^{k} \widetilde{R}_{y^{+}}(y)=\partial^{k} \widetilde{R}_{y^{-}}(y), \quad k=0,1, \cdots, 10,
$$

and

$$
\partial^{11} \widetilde{R}_{y^{+}}(y)-\partial^{11} \widetilde{R}_{y^{-}}(y)=1 .
$$

The unknown coefficients $c_{\mathfrak{i}}(y)$ and $d_{\mathfrak{i}}(y)(\mathfrak{i}=1,2, \cdots, 10)$ can be obtained from (2.2), (2.3), (2.4), (2.5), (2.6). This completes the proof.

Definition 2.3. The space $\widehat{W}_{2}^{1}[-1,1]$ is defined by

$$
\widehat{W}_{2}^{1}[-1,1]=\left\{u \in A C[-1,1]: u^{\prime} \in \mathrm{L}^{2}[-1,1]\right\} .
$$

The inner product and the norm in $\widehat{W}_{2}^{1}[-1,1]$ are obtained as

$$
\langle v, u\rangle_{\widehat{W}_{2}^{1}}=v(-1) u(-1)+\int_{-1}^{1} v^{\prime}(x) u^{\prime}(x) d x, \quad v, u \in \widehat{W}_{2}^{1}[-1,1]
$$

and

$$
\|v\|_{\widehat{W}_{2}^{1}}=\sqrt{\langle v, v\rangle \widehat{W}_{2}^{1}}, \quad v \in \widehat{W}_{2}^{1}[-1,1]
$$

Theorem 2.4. The space $\widehat{W}_{2}^{1}[-1,1]$ is a reproducing kernel space, and its reproducing kernel function $\widetilde{\mathrm{Q}_{y}}$ is obtained

$$
\widetilde{Q_{y}}(x)= \begin{cases}2+x, & -1 \leqslant x \leqslant y \leqslant 1, \\ 2+y, & -1 \leqslant y<x \leqslant 1 .\end{cases}
$$

Proof. The proof of Theorem 2.4 is similar to the proof of Theorem 2.2. Therefore the proof is omitted.

3. Solution representation in $\widehat{\mathbf{W}}_{2}^{6}[-1,1] \oplus \widehat{\mathbf{W}}_{2}^{6}[-1,1]$

Lemma 3.1. If $B_{i j}: \widehat{W}_{2}^{6}[-1,1] \rightarrow \widehat{W}_{2}^{1}[-1,1], i, j=1,2$, are bounded linear operators, then

$$
\mathrm{B}: \widehat{\mathrm{W}}_{2}^{6}[-1,1] \oplus \widehat{\mathrm{W}}_{2}^{6}[-1,1] \rightarrow \widehat{\mathrm{W}}_{2}^{1}[-1,1] \oplus \mathrm{W}_{2}^{1}[-1,1],
$$

is a bounded linear operator. 
Proof. We get

$$
\begin{aligned}
\|\mathrm{Bu}\| & =\left(\sum_{i=1}^{2}\left\|\sum_{j=1}^{2} \mathrm{~B}_{i j} v_{j}\right\|^{2}\right)^{\frac{1}{2}} \\
& \leqslant\left[\sum_{i=1}^{2}\left(\sum_{j=1}^{2}\left\|\mathrm{~B}_{i j}\right\|\left\|v_{j}\right\|\right)^{2}\right]^{\frac{1}{2}} \\
& \leqslant\left[\sum_{i=1}^{2}\left(\sum_{j=1}^{2}\left\|\mathrm{~B}_{i j}\right\|^{2}\right)\left(\sum_{j=1}^{2}\left\|v_{j}\right\|^{2}\right)\right]^{\frac{1}{2}} \\
& =\left(\sum_{i=1}^{2} \sum_{j=1}^{2}\left\|\mathrm{~B}_{i j}\right\|^{2}\right)^{\frac{1}{2}}\|v\| .
\end{aligned}
$$

$B$ is bounded by the boundedness of $B_{i j}$. This completes the proof.

Now, put

$$
\varphi_{i j}(x)=\widetilde{Q}_{x_{i}}(x) \overrightarrow{e_{j}}= \begin{cases}\left(\widetilde{Q}_{x_{i}}(x), 0\right)^{\top}, & j=1, \\ \left(0, \widetilde{Q}_{x_{i}}(x)\right)^{\top}, & j=2,\end{cases}
$$

and $\psi_{i j}(x)=B^{*} \varphi_{i j}(x), i=1,2, \cdots, j=1,2$, where $B^{*}$ is the conjugate operator of $B$. The orthonormal system of $\left\{\widehat{\psi}_{i j}(x)\right\}_{(1,1)}^{(\infty, 2)}$ of $\widehat{W}_{2}^{6}[-1,1] \oplus \widehat{W}_{2}^{6}[-1,1]$ can be obtained as

$$
\widehat{\psi}_{i j}(x)=\sum_{z=1}^{i} \sum_{q=1}^{j} \beta_{z q}^{i j} \psi_{z q}(x), \quad i=1,2, \cdots, j=1,2 .
$$

Theorem 3.2. Assume $\left\{x_{i}\right\}_{i=1}^{\infty}$ is dense in $[-1,1]$. Then $\left\{\psi_{i j}(x)\right\}_{(1,1)}^{(\infty, 2)}$ is a complete system in

$$
\widehat{W}_{2}^{6}[-1,1] \oplus \widehat{W}_{2}^{6}[-1,1] \text {. }
$$

Proof. Let $\left\langle v(x), \Psi_{i j}(x)\right\rangle=0(i=1,2, \cdots)$, for each fixed $v(x) \in \widehat{W}_{2}^{6}[-1,1] \oplus \widehat{W}_{2}^{6}[-1,1]$. We have

$$
\left\langle\mathrm{B} v(x), \varphi_{i j}(x)\right\rangle=0 .
$$

Note that

$$
v(x)=\sum_{j=1}^{2} v_{j}(x) \overrightarrow{e_{j}}=\sum_{j=1}^{2}\left\langle v(.), R_{x}(.) \overrightarrow{e_{j}}\right\rangle \overrightarrow{e_{j}}
$$

Thus,

$$
\mathrm{B} v\left(x_{i}\right)=\sum_{j=1}^{2}\left\langle\mathrm{~B} v(y), \varphi_{i j}(y)\right\rangle \overrightarrow{e_{j}}=0, \quad(i=1,2, \cdots),
$$

by (3.1). We obtain $(B v)(x)=0$. We acquire $v \equiv 0$ by B $^{-1}$. As a result, $\left\{\psi_{i j}(x)\right\}_{(1,1)}^{(\infty, 2)}$ is a complete system in $\widehat{W}_{2}^{6}[-1,1] \oplus \widehat{W}_{2}^{6}[-1,1]$. This completes the proof. 


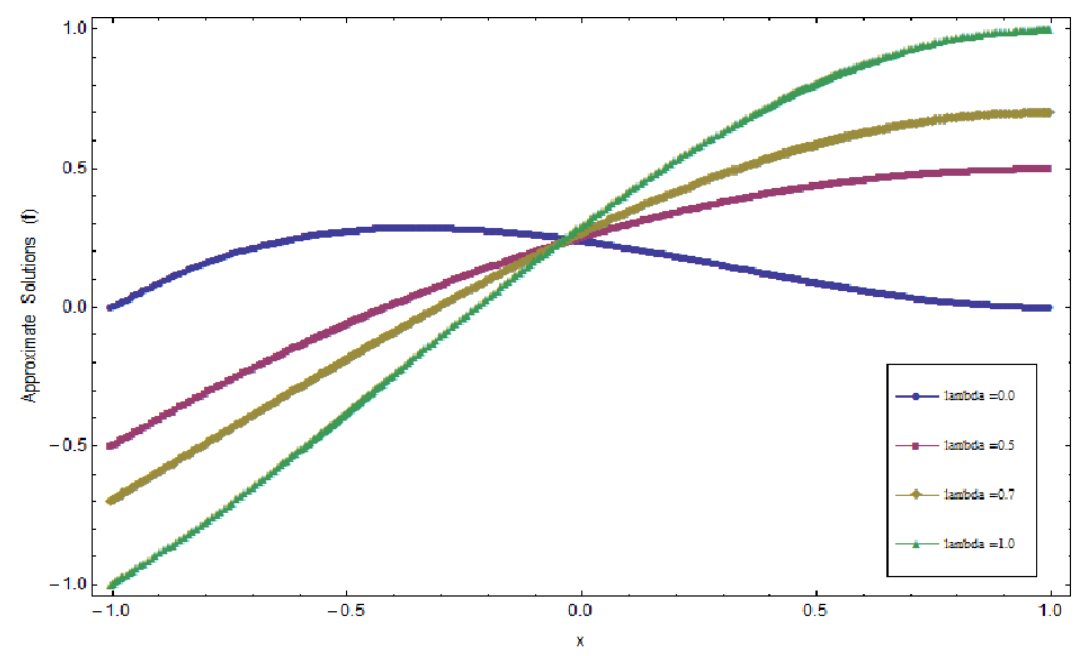

Figure 1: Approximate solutions of $f$ for $M=0.1, \alpha=0.2, K=0.1$, and $R=0.2$.

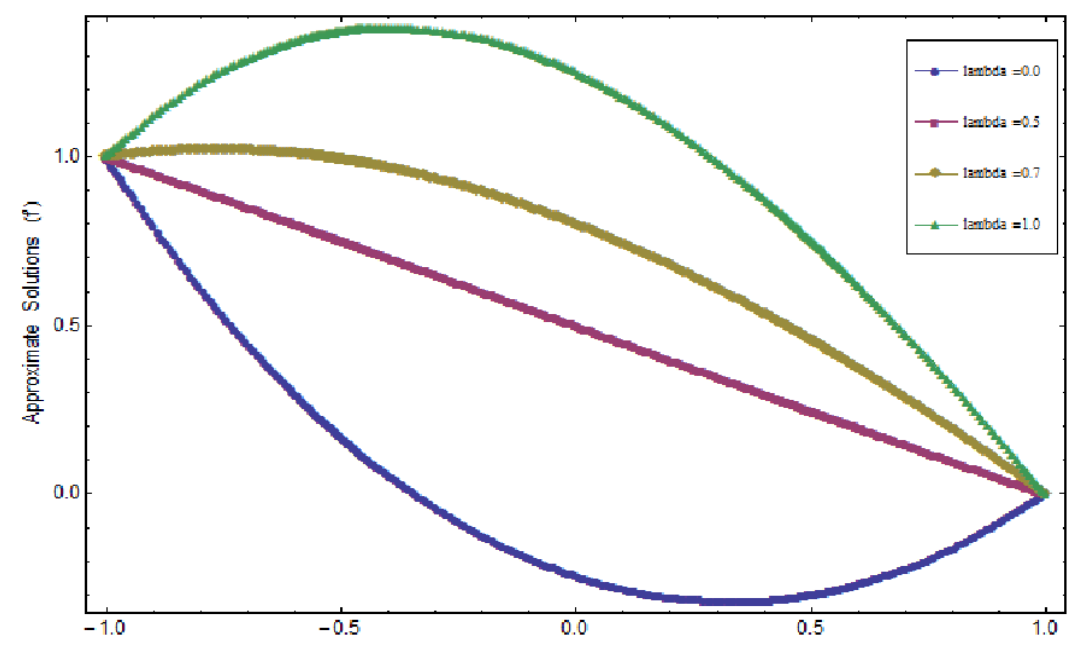

Figure 2: Approximate solutions of $\mathrm{f}^{\prime}$ for $M=0.1, \alpha=0.2, \mathrm{~K}=0.1$, and $\mathrm{R}=0.2$.

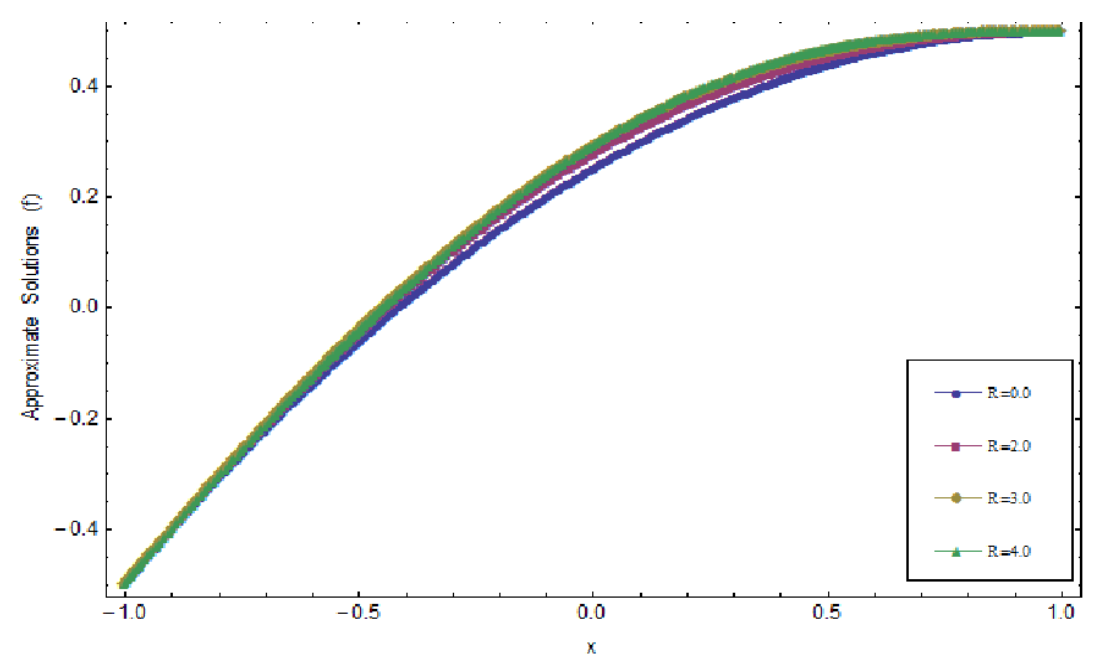

Figure 3: Approximate solutions of $f$ for $M=0.1, K=0.2, \lambda=0.5$, and $\alpha=0.2$. 


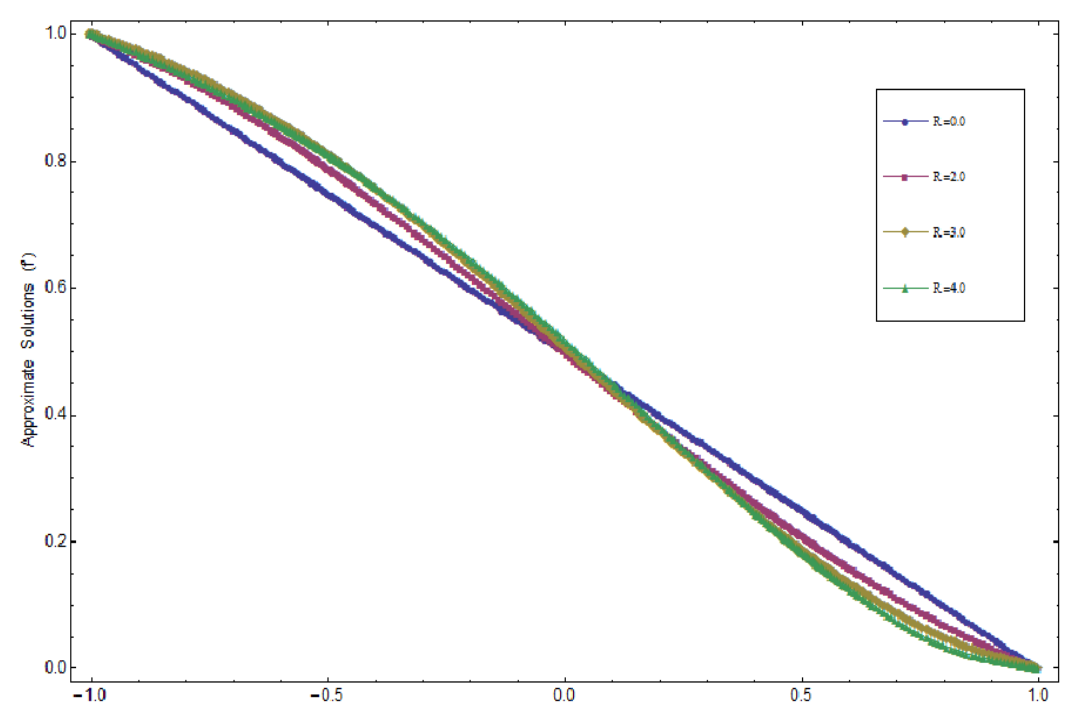

Figure 4: Approximate solutions of $\mathrm{f}^{\prime}$ for $M=0.1, \mathrm{~K}=0.2, \lambda=0.5$, and $\alpha=0.2$.

Theorem 3.3. If $\left\{x_{i}\right\}_{i=1}^{\infty}$ is dense in $[-1,1]$, then the solution of (1.2) satisfies

$$
v=\sum_{i=1}^{\infty} \sum_{j=1}^{2} \sum_{z=1}^{i} \sum_{q=1}^{j} \beta_{z q}^{i j} M\left(x_{z}, h\left(x_{z}\right), p\left(x_{z}\right)\right) .
$$

Proof. Let $v$ be the solution of (1.2). By Theorem 3.2, $\left\{\Psi_{i j}(x)\right\}_{(1,1)}^{(\infty, 2)}$ is the complete orthonormal basis of $\widehat{W}_{2}^{6}[-1,1] \oplus \widehat{W}_{2}^{6}[-1,1]$. Therefore

$$
\begin{aligned}
v & =\sum_{i=1}^{\infty} \sum_{j=1}^{2}\left\langle v(x), \widehat{\psi}_{i j}(x)\right\rangle \widehat{\psi}_{i j}(x) \\
& =\sum_{i=1}^{\infty} \sum_{j=1}^{2}\left\langle v(x), \sum_{z=1}^{i} \sum_{q=1}^{j} \beta_{z q}^{i j} \widehat{\psi}_{z q}(x)\right\rangle \widehat{\psi}_{i j}(x) \\
& =\sum_{i=1}^{\infty} \sum_{j=1}^{2} \sum_{z=1}^{i} \sum_{q=1}^{j} \beta_{z q}^{i j}\left\langle v(x), B^{*} \varphi_{z q}(x)\right\rangle \widehat{\psi}_{i j}(x) \\
& =\sum_{i=1}^{\infty} \sum_{j=1}^{2} \sum_{z=1}^{i} \sum_{q=1}^{j} \beta_{z q}^{i j}\left\langle B v(x), \varphi_{z q}(x)\right\rangle \widehat{\psi}_{i j}(x) \\
& =\sum_{i=1}^{\infty} \sum_{j=1}^{2} \sum_{z=1}^{i} \sum_{q=1}^{j} \beta_{z q}^{i j} M\left(x_{z}, h\left(x_{z}\right), p\left(x_{z}\right)\right) \widehat{\psi}_{i j}(x) .
\end{aligned}
$$

The approximate solution $v_{n}$ can be acquired from the n-term intercept of the exact solution $v$ and

$$
v_{n}=\sum_{i=1}^{n} \sum_{j=1}^{2} \sum_{z=1}^{i} \sum_{q=1}^{j} \beta_{z q}^{i j} M\left(x_{z}, h\left(x_{z}\right), p\left(x_{z}\right)\right) .
$$




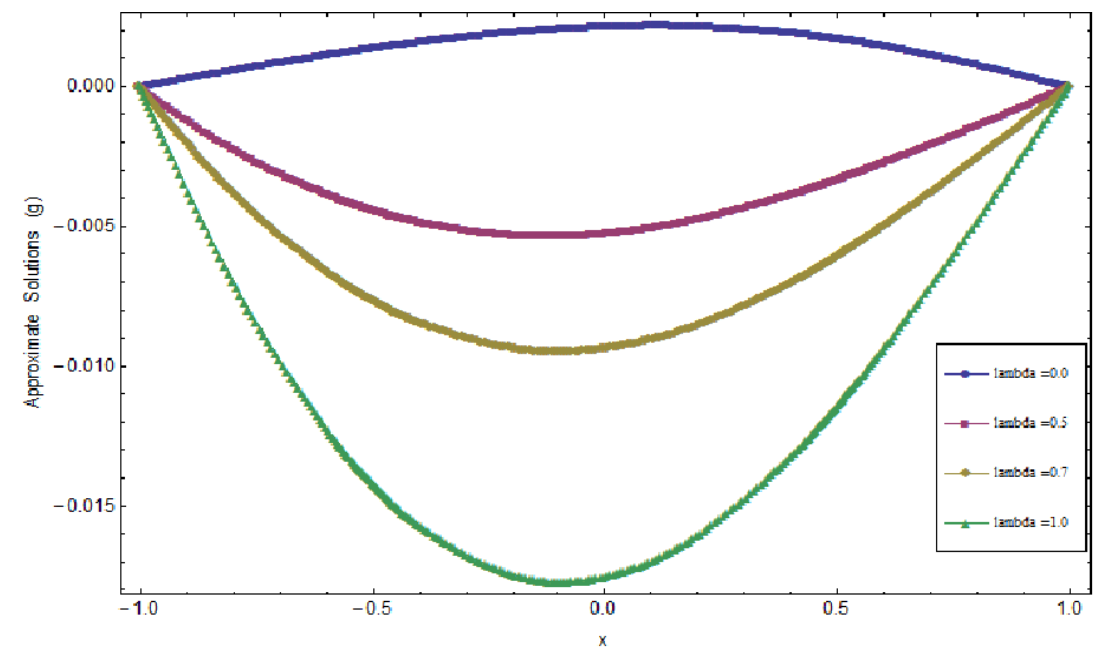

Figure 5: Approximate solutions of $g$ for $M=0.1, K=0.1, \alpha=0.2$, and $R=0.2$.

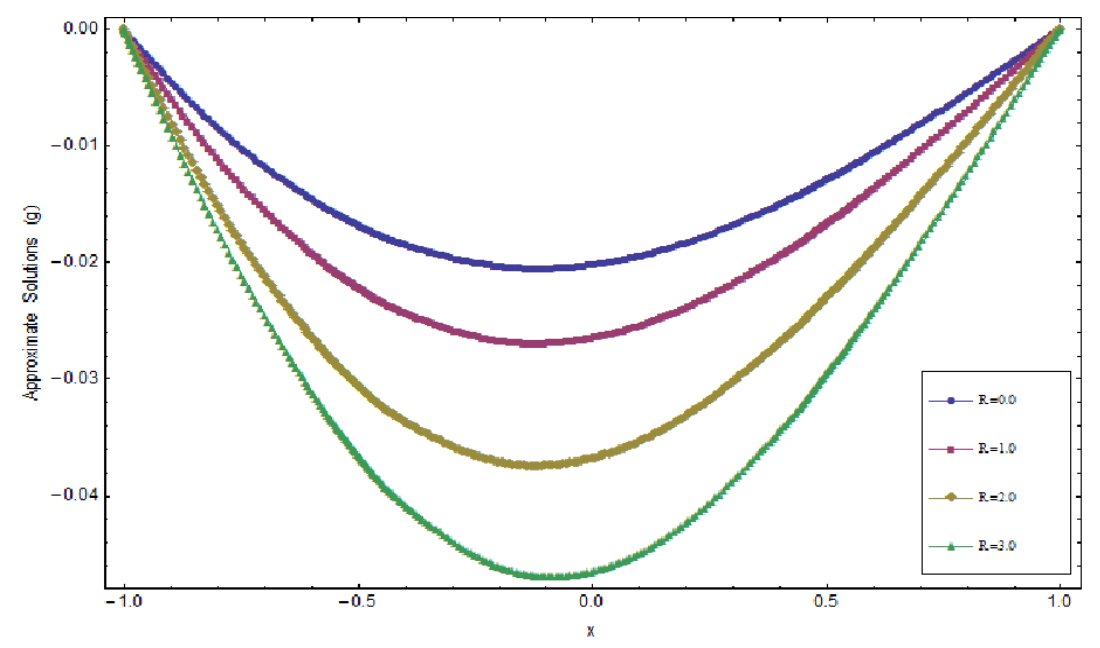

Figure 6: Approximate solutions of $g$ for $\lambda=0.5, K=0.2, \alpha=0.2$, and $M=0.1$.

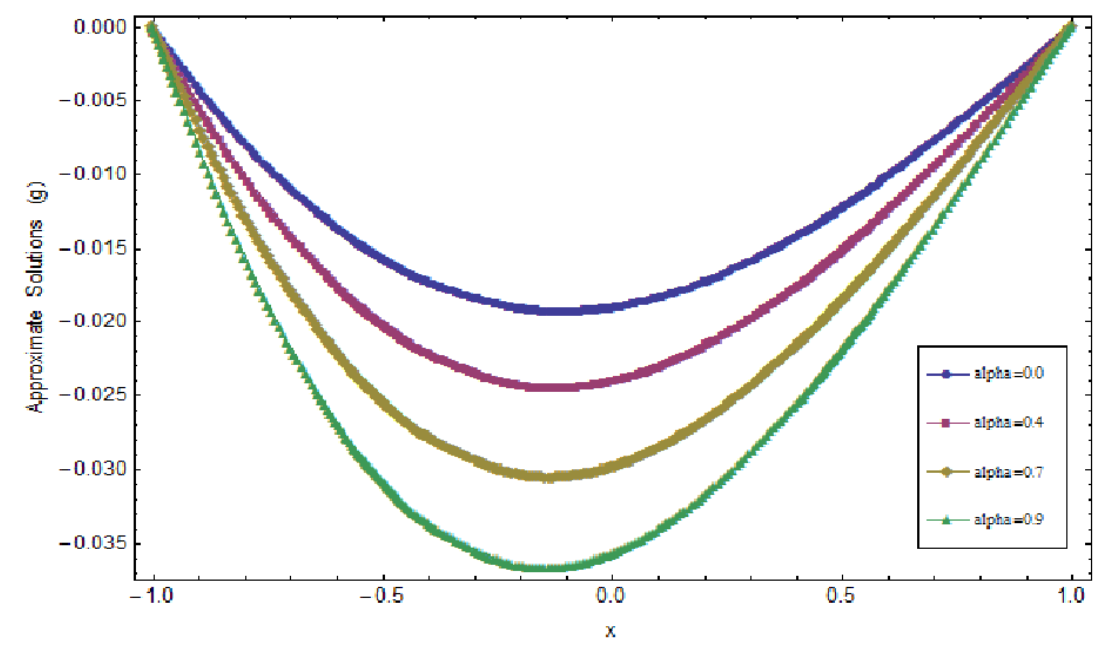

Figure 7: Plots approximate solutions of $g$ for $\lambda=0.5, K=0.2, M=0.1$, and $R=0.2$. 


\section{Numerical results}

We conceive the following nonlinear system in the reproducing kernel space in this section.

$$
\left\{\begin{array}{l}
f^{(i v)}-M^{2} f^{\prime \prime}+2 K g^{\prime}+R\left(f^{\prime} f^{\prime \prime}-f f^{\prime \prime \prime}\right)-\alpha f f^{(v)} \\
\quad-3 \alpha f^{\prime \prime} f^{\prime \prime \prime}-\alpha f^{\prime} f^{(i v)}-\alpha g g^{\prime \prime \prime}+\alpha\left(f^{\prime \prime}\right)^{2}+\alpha g^{\prime} g^{\prime \prime}=0, \\
g^{\prime \prime}-M^{2} g-2 K^{2} f^{\prime}+R\left(f^{\prime} g-f g^{\prime}\right)+\alpha f g^{\prime \prime \prime}-\alpha f^{\prime} g^{\prime \prime}=0, \\
f=-\lambda, \quad f^{\prime}=1, \quad g=0, \quad \text { at } \eta=-1, \\
f=\lambda, \quad f^{\prime}=0, \quad g=0, \quad \text { at } \eta=1,
\end{array}\right.
$$

where $M, K, \alpha, R$ and $\lambda$ are constants.

After homogenizing the conditions we acquired the numerical results of (4.1) for different values of $M, K, \alpha, R$ and $\lambda$ and demonstrated them by Figures $1-7$.

\section{Conclusion}

We investigated approximate solutions of nonlinear systems in the reproducing kernel space in this work. We presented our results with Figures 1-7. We verified that reproducing kernel method is very effective technique for solving nonlinear systems.

\section{Acknowledgment}

The authors extend their appreciation to the International Scientific Partnership Program ISPP at King Saud University for funding this research work through ISPP 63.

\section{References}

[1] G. T. Adams, N. S. Feldman, P. J. McGuire, Tridiagonal reproducing kernels and subnormality, J. Operator Theory, 70 (2013), 477-494. 1

[2] G. Akram, H. U. Rehman, Numerical solution of eighth order boundary value problems in reproducing kernel space, Numer. Algorithms, 62 (2013), 527-540.

[3] G. Akram, H. U. Rehman, Solutions of a class of sixth order boundary value problems using the reproducing kernel space, Abstr. Appl. Anal., 2013 (2013), 8 pages.

[4] G. Akram, H. U. Rehman, Solution of the system of fourth order boundary value problem using reproducing kernel space, J. Appl. Math. Inform., 31 (2013), 55-63. 1

[5] N. Aronszajn, Theory of reproducing kernels, Trans. Amer. Math. Soc., 68 (1950), 337-404. 1

[6] O. A. Arqub, M. Al-Smadi, N. Shawagfeh, Solving Fredholm integro-differential equations using reproducing kernel Hilbert space method, Appl. Math. Comput., 219 (2013), 8938-8948. 1

[7] A. Baranov, Y. Belov, A. Borichev, Hereditary completeness for systems of exponentials and reproducing kernels, Adv. Math., 235 (2013), 525-554. 1

[8] A. H. Bhrawy, M. A. Abdelkawy, E. M. Hilal, A. A. Alshaery, A. Biswas, Solitons, cnoidal waves, snoidal waves and other solutions to Whitham-Broer-Kaup system, Appl. Math. Inf. Sci., 8 (2014), 2119-2128. 1

[9] A. H. Bhrawy, J. F. Alzaidy, M. A. Abdelkawy, A. Biswas, Jacobi spectral collocation approximation for multidimensional time-fractional Schrdinger equations, 84 (2016), 1553-1567.

[10] A. Biswas, Solitary wave solution for KdV equation with power-law nonlinearity and time-dependent coefficients, Nonlinear Dynam., 58 (2009), 345-348. 1

[11] D. Biswas, T. Banerjee, A simple chaotic and hyperchaotic time-delay system: design and electronic circuit implementation, Nonlinear Dynam., 83 (2016), 2331-2347. 1

[12] S. Bushnaq, S. Momani, Y. Zhou, A reproducing kernel Hilbert space method for solving integro-differential equations of fractional order, J. Optim. Theory Appl., 156 (2013), 96-105. 1

[13] L. P. Castro, S. Saitoh, Optimal and approximate solutions of singular integral equations by means of reproducing kernels, Complex Anal. Oper. Theory, 7 (2013), 1839-1851. 
[14] S.-W. Chi, J.-S. Chen, H.-Y. Hu, J. P. Yang, A gradient reproducing kernel collocation method for boundary value problems, Internat. J. Numer. Methods Engrg., 93 (2013), 1381-1402. 1

[15] G. Ebadi, A. Biswas, Application of $\mathrm{G}^{\prime} / \mathrm{G}$-expansion method to Kuramoto-Sivashinsky equation, Acta Math. Appl. Sin. Engl. Ser., 32 (2016), 623-630. 1

[16] A. G. García, A. Portal, Sampling in reproducing kernel Banach spaces, Mediterr. J. Math., 10 (2013), 1401-1417. 1

[17] F.-Z. Geng, M.-G. Cui, Solving a nonlinear system of second order boundary value problems, J. Math. Anal. Appl., 327 (2007), 1167-1181. 1

[18] F.-Z. Geng, M.-G. Cui, A reproducing kernel method for solving nonlocal fractional boundary value problems, Appl. Math. Lett., 25 (2012), 818-823. 1

[19] F.-Z. Geng, S. P. Qian, Reproducing kernel method for singularly perturbed turning point problems having twin boundary layers, Appl. Math. Lett., 26 (2013), 998-1004. 1

[20] M. Inc, E. Fendoglu, H. Triki, A. Biswas, Compactons and topological solitons of the Drinfel'd-Sokolov system, Nonlinear Anal. Model. Control, 19 (2014), 209-224. 1

[21] Y. Jia, Y.-J. Zhang, G. Xu, X.-Y. Zhuang, T. Rabczuk, Reproducing kernel triangular B-spline-based FEM for solving PDEs, Comput. Methods Appl. Mech. Engrg., 267 (2013), 342-358. 1

[22] W. Jiang, Z. Chen, Solving a system of linear Volterra integral equations using the new reproducing kernel method, Appl. Math. Comput., 219 (2013), 10225-10230.

[23] M. T. Karaev, Erratum: Use of reproducing kernels and Berezin symbols technique in some questions of operator theory [Forum Math., DOI 10.1515/FORM.2011.073] [ MR2926635], Forum Math., 25 (2013), 1107.

[24] X.-Y. Li, B.-Y. Wu, Reproducing kernel method for singular multi-point boundary value problem, Math. Sci. (Springer), 6 (2012), 5 pages.

[25] X.-Y. Li, B.-Y. Wu, New algorithm for nonclassical parabolic problems based on the reproducing kernel method, Math. Sci. (Springer), 7 (2013), 5 pages.

[26] M. Mohammadi, R. Mokhtari, H. Panahipour, A Galerkin-reproducing kernel method: application to the 2D nonlinear coupled Burgers' equations, Eng. Anal. Bound. Elem., 37 (2013), 1642-1652.

[27] J. Niu, Y.-Z. Lin, M.-G. Cui, A novel approach to calculation of reproducing kernel on infinite interval and applications to boundary value problems, Abstr. Appl. Anal., 2013 (2013), 7 pages.

[28] K. Özen, K. Oruçoğlu, Approximate solution to a multi-point boundary value problem involving nonlocal integral conditions by reproducing kernel method, Math. Model. Anal., 18 (2013), 529-536. 1

[29] K. R. Rajagopal, On boundary conditions for fluids of the differential type, Navier-Stokes equations and related nonlinear problems, Funchal, (1994), Plenum, New York, 278 (1995), 273-278. 1

[30] B.-H. Sheng, P.-X. Ye, The learning rates of regularized regression based on reproducing kernel Banach spaces, Abstr. Appl. Anal., 2013 (2013), 10 pages. 1

[31] S. B. Sontz, A reproducing kernel and Toeplitz operators in the quantum plane, Commun. Math., 21 (2013), 137-160.

[32] S. Twareque Ali, F. Bagarello, J. Pierre Gazeau, Quantizations from reproducing kernel spaces, Ann. Physics, 332 (2013), 127-142.

[33] Y.-L. Wang, T. Chaolu, Z. Chen, Using reproducing kernel for solving a class of singular weakly nonlinear boundary value problems, Int. J. Comput. Math., 87 (2010), 367-380.

[34] Y.-L. Wang, S. Lu, F.-G. Tan, M.-J. Du, H. Yu, Solving a class of singular fifth-order boundary value problems using reproducing kernel Hilbert space method, Abstr. Appl. Anal., 2013 (2013), 6 pages. 1

[35] Y.-L. Wang, L.-J. Su, X.-J. Cao, X.-N. Li, Using reproducing kernel for solving a class of singularly perturbed problems, Comput. Math. Appl., 61 (2011), 421-430. 1

[36] W.-Y. Wang, M. Yamamoto, B. Han, Numerical method in reproducing kernel space for an inverse source problem for the fractional diffusion equation, Inverse Problems, 29 (2013), 15 pages. 1

[37] B. Y. Wu, X. Y. Li, A new algorithm for a class of linear nonlocal boundary value problems based on the reproducing kernel method, Appl. Math. Lett., 24 (2011), 156-159. 1

[38] S.-S. Xie, S.-Y. Heo, S.-C. Kim, G.-S. Woo, S.-C. Yi, Numerical solution of one-dimensional Burgers' equation using reproducing kernel function, J. Comput. Appl. Math., 214 (2008), 417-434. 1

[39] L.-H. Yang, H.-Y. Li, J.-R. Wang, Solving a system of linear Volterra integral equations using the modified reproducing kernel method, Abstr. Appl. Anal., 2013 (2013), 5 pages. 1

[40] H.-M. Yao, Y.-Z. Lin, Solving singular boundary-value problems of higher even-order, J. Comput. Appl. Math., 223 (2009), 703-713. 1

[41] Y. F. Zhou, X. Q. Lü, Y. Y. Zhang, Solving a class of integral equations in a reproducing kernel space, (Chinese) Natur. Sci. J. Harbin Normal Univ., 22 (2006), 12-15. 1 